\title{
Naiver Realismus? Zur Gegenständlichkeit des Sammelns
}

\section{Wann ist eine Kuh nichts als eine Kuh?}

Stellen wir uns für einen Moment eine weidende Kuh vor, die gemeinsam mit Artgenossen grast. Unser Weltwissen erlaubt uns, sie einer Herde zuzuordnen (was möglicherweise gar nicht den Tatsachen entspricht). Würden wir die Herde eine ,Sammlung‘ von Kühen nennen? Laut der relevanten Definition aus dem Grimm'schen Wörterbuch bezeichnet die Sammlung „eine nach bestimmten gesichtspunkten wissenschaftlicher, künstlerischer zwecke oder der liebhaberei zusammengebrachte und geordnete menge von gegenständen“ (Deutsches Wörterbuch 1854ff. [Lemma: „Sammlung“]). Weder ist die Integrität das Prinzip der Bewahrung einer Kuhherde noch Selbstzweck Prinzip ihrer Funktion. Selbst eine Herde, die unter wissenschaftlicher Anleitung und unabhängig von Milch- oder Fleischproduktion zusammengestellt wurde (etwa im Hinblick auf genetische Studien), wäre in unserem Sprachgebrauch keine Sammlung. Die Kuh wäre folglich auch kein Sammlerobjekt.

Stellen wir uns nun das Gemälde einer Kuh vor, beispielsweise die Liegende Kuh (1883) von Vincent van Gogh. Wir könnten sagen, dass diese einzelne Kuh Teil einer Sammlung sei, allerdings eben einer Gemäldesammlung. Nicht die Kuh, sondern ihre Abbildung gehört in die Sammlung, wobei über den Charakter der Sammlung damit noch nichts ausgesagt ist. Es könnte sich um eine Sammlung nur von Tierporträts handeln oder gar um eine Sammlung, die sich allein auf die Repräsentationen von Nutztieren spezialisiert. Es könnte sich freilich auch um eine Sammlung niederländischer Malerei handeln, um einen reinen Van Gogh-Bestand usf.: Sammlungen müssen offenbar implizit oder explizit die Kriterien mitliefern, die zu ihrer Konstitution geführt haben, sonst können sie nicht als solche erkannt werden.

Letzter Fall: wir befinden uns in einer Ausstellung, in der eine echte, ehemals lebendige Kuh (und nicht nur ihr Abbild) ausgestellt wird. Ich denke etwa an Damien Hirsts berühmte, in Formaldehyd eingelegte und längs geteilte Kuh aus dem Kunstwerk Mother and Child, Divided (1993; heute im Astrup Fearnley Museum Oslo). Hier haben wir es tatsächlich mit einer Kuh zu tun, die Teil einer Sammlung ist. Indes wäre die Erkenntnis, dass es sich bei dem Gegenstand um eine Kuh handelt, unterkomplex und wenig hilfreich für eine anspruchsvollere Aneignung des Werks. Damien Hirsts Kuh als bloße Kuh zu identifizieren, wäre 
eine kindliche Reaktion. Aber selbst ein Kind, zumal ein aufgewecktes, würde sofort beginnen, weitergehende Frage zu stellen. Warum sind Kuh und Kalb in der Mitte durchgesägt? Können sie wieder lebendig werden? Wer hat sie hergebracht? Es gibt keinen notwendigen Zusammenfall zwischen den Intentionen, die zur Sammlung führten, und dem Umgang mit ihnen.

Diese beliebigen Beispiele sollen eine Reihe von Grundproblemen offenlegen, die hinter der Frage nach den Logiken der Sammlung stehen. Ausgehend von der oben zitierten Wörterbuchdefinition könnte man sagen, die Logik der Sammlung ist entweder eine Epistemo-Logik, d. h. eine Logik der „gesichtspunkte“, unter denen die Sammlung zustandekam, oder eine Onto-Logik, d. h. eine Logik der „gegenstände“ selbst. Im ersten Fall interessiere ich mich für die Bedingungen der Möglichkeit der Sammlung, im zweiten für die Einheiten, aus denen sie besteht, vielleicht sogar für die Bezüge, die sie ausbilden. Es besteht also ein wesentlicher Unterschied darin, ob ich den Bestimmungsgrund der Sammlung vornehmlich vom Subjekt bzw. Urheber der Sammlung oder aber von den Objekten bzw. ihrer Eigenlogik ableite. Es sollte auf der Hand liegen, dass beide Seiten berücksichtigt werden müssen, um der Komplexität von Sammlungen gerecht zu werden. In der Praxis ist dies aber meistens nicht der Fall; hier wie auf anderen Gebieten auch herrscht geradezu ein Schisma zwischen epistemologischen und ontologischen Orientierungen. Ich möchte deshalb mit einer Theorie der „Gegenständlichkeit“ eine Alternative anbieten, die von der dialektischen Verschränkung epistemologischer, ontologischer und (im weitesten Sinne) pragmatischer Perspektiven ausgeht. Ich interessiere mich dafür, wie die Logiken der Sammlungen in der Bestimmung ihrer Gegenstände zusammenfallen. Es reicht nicht aus, eine Kuh als Gegenstand zu identifizieren, ohne nach den Praktiken zu fragen, die sie als Sammlungsgegenstand legitimieren oder ausschließen. Umgekehrt reicht die Vertrautheit mit den Praktiken nicht aus, um die Aufnahme einer Kuh (und nicht etwa eines anderen Säugetiers) in die jeweilige Sammlung zu verstehen.

\section{Kritik des vortheoretischen Sammelns als Metaphysikkritik}

Der Landwirt, der von Milchwirtschaft lebt, wird sich den eingangs gestellten Fragen eher selten ausgesetzt sehen. Die Bedingungen der Möglichkeit von, sagen wir, ,Kuhheit‘ lassen die Jungbäuerin aus nachvollziehbaren Gründen kalt. Es gibt Bereiche, in denen eine klare und eindeutige Trennung der Sphären - Landwirt hier, Kreatur da - nicht nur ausreichend, sondern den Abläufen, um die es geht, angemessen ist. Auf dem Gebiet der Sammlungen wäre an Briefmarkenoder Bierdeckelsammlungen zu denken, bei denen weder die Bestimmung des Sammlersubjekts noch des Sammlerobjekts unüberwindliche Herausforderun- 
gen zeitigt. Wir können solche Sammlungen als vortheoretische bezeichnen, weil weder ihre Epistemologie noch ihre Ontologie bewusst reflektiert wird oder reflektiert werden muss, sondern sich aus tradierten Praktiken von selbst ergibt. Eine vortheoretische Sammlung interessiert sich für die vom unproblematischen Sammlersubjekt unabhängigen Sammlungsobjekte. Diese Sammlungen verfolgen zumeist einen Vollständigkeitsanspruch bzw. sind, im Sinne einer intuitiven Mengenlehre, auf die zumindest theoretisch mögliche Vollständigkeit angelegt (alle Briefmarken einer bestimmten Serie usw.).

Vielleicht entsprechen die meisten Sammlungen, die wir kennen, diesem Typ. Ohne sie gäbe es die Schätze nicht, die sich in Museen, Archiven oder Privaträumen über die ganze Welt verteilt finden. Sie haben wesentlich zur Herausbildung der Welt beigetragen, in der wir leben. Eine Kritik der vortheoretischen Sammlung fällt deshalb zwangsläufig mit einer Kritik des Bestehenden zusammen, ist womöglich der Anlass dazu. Genau diesen Fall verkörpert Martin Heideggers Denken des Sammelns. Heidegger ist einer der wenigen Philosophen, die sich explizit an einem solchen Denken versucht haben. ${ }^{1}$ Heidegger möchte einen neuen Begriff des Sammelns entwickeln, der dem herkömmlichen entgegengesetzt ist:

Wir sollen nur das Augenmerk darauf richten, daß das bloße Sammeln, der sogenannte museale Betrieb, ohne die Gesammeltheit des geschichtlichen Menschen auf die innere Versammlung und Wahrung seines Wesens kein wahrhaftes Sammeln ist, daß somit das Wesen des Sammelns keineswegs im aufraffenden Beibringen und Ausstellen sich erschöpft. (Heidegger 1975ff., GA 55, 291)

Der gewöhnliche Ausstellungsbetrieb wird hier von einem noch näher zu erläuternden ,wahrhaften' Sammeln abgesetzt, das auf (vorerst nebulöse) Weise mit dem ,Wesen“ des Menschen zusammenhängt. Dieses Sammeln ist abhängig von einem „Bestimmungsgrund“, der auf das „Aufbewahren und Bewahren“ hin orientiert ist, mithin nicht um den Besitz der Sammelobjekte um des Besitzes (,aufraffend') noch um des bloß äußerlichen Zurschaustellens willen, sondern weil die Sammlung den Grund ihres Willens zum Bewahren schon in sich enthält. Sie bedarf deshalb eines ,schon waltenden und konzentrierenden Zentrum[s]“, des Logos, der „das alles vereinende Eine“ und zugleich die „abwesende Gegenwart“ sei - also das Sein am Grunde des Seienden (Heidegger 1975ff., GA 55, 269, 317).

Diese Analyse hängt zusammen mit Heideggers Auffassung des Lesens als Lese, d. h. als Sammlung. Heidegger möchte das Lesen aus dem Bereich der „Rede

1 Siehe aber Sommer (1999) sowie Skirl et al. (2000). Das Denken des Sammelns spielt in soziologischen und anthropologischen Ansätzen eine wichtigere Rolle (z. B. Stagl 1998). 
und Sprache“ (Heidegger 1975ff., GA 55, 266) herauslösen, also von einem subjektzentrierten Menschenbild emanzipieren und über den Umweg des légein hin zum sammelnden und versammelnden Aufnehmen verschieben: „Das eigentliche Lesen ist die Sammlung auf das, was ohne unser Wissen einst schon unser Wesen in den Anspruch genommen hat" (Heidegger 1975ff., GA 13, 111). Es ist die Aufbewahrung, in der das Zerstreute wieder zusammengeführt wird (Heidegger 1975ff., GA 55, 267-269). Das wahre Sammeln unterscheidet sich für Heidegger von dem kritisierten mit anderen Worten dadurch, dass an die Objekte keine ihnen äußerlichen, rein subjektiven Kriterien herangetragen werden. Im Gegenteil tritt der Sammelnde hinter die Sammlung zurück, geht in ihr auf. Logik ist immer schon eine Sammlung, umgekehrt beruht eine Sammlung auf einer Logik, die in Wahrheit auf dem Sein aufruht.

Heideggers Einlassungen können in unterschiedliche Richtungen weiterentwickelt werden. Durchgesetzt hat sich die Ablehnung der scharfen Trennung zwischen einem nicht näher problematisierten Subjekt und dem allenfalls erkenntnistheoretisch problematisierten Objekt, dessen In-der-Welt-Sein dadurch vernachlässigt wird. Die Sammlung, die von sich selber glaubt, schlichte Anordnung einer individuellen Sammelpraxis zu sein, wird zum Emblem einer Einteilung der Welt nach metaphysischen Grundsätzen, die weder philosophischer Fundamentalkritik noch naturwissenschaftlichen Einsichten standhielte. Nicht durchgesetzt hat sich, wohl v. a. aus pragmatischen Gründen, die Abschaffung des Sammlersubjekts, um die es eigentlich ging. Das Sammeln wird demzufolge theoretisch erst mit den letzten Resten der Moderne obsolet werden, die so lange unvollendet ist, wie sie noch am Subjekt festhält.

\section{Realismusproblem und Materialitätsdebatte: Welcher Realismus darf es sein?}

Heideggers von Modernefeindschaft und Zivilisationskritik getragene Philosophie des Sammelns enthält nicht nur eine allgemeine Subjekt- und Metaphysikkritik, sondern notwendigerweise auch eine spezifische Kritik am metaphysischen Realismus. Denn nur ihm lässt sich das naive, vortheoretische Sammeln zuordnen. Dass Sammlungen allerdings irgendein Verhältnis zum Realismus entwickeln müssen, folgt zwingend schon aus der konstitutiven Rolle der Sammelgegenstände für jede Sammlung. Doch welche Alternativen gibt es überhaupt zum metaphysischen Realismus? Der metaphysische Realismus ist jedenfalls, nicht nur wegen Heidegger und seiner Folgen zumal in den Geisteswissenschaften, lange die unpopulärste Version des Realismus gewesen; man trifft ihn neuerdings freilich dort wieder an, wo der Wunsch, die Geisteswissenschaften auf ein 
vermeintlich solides empirisches Fundament zu stellen, mit einer Ablehnung der avancierten theoretischen Reflexionen der letzten Jahrzehnte einhergeht. ${ }^{2}$ Dem reduktiven Weltverständnis des metaphysischen Realismus gelten nur diejenigen Objekte als relevant, die eindeutig voneinander abgrenzbar und frei von subjektiver Einmischung analysierbar sind. Ihm entsprechen typischerweise Ontologien der „moderate sized specimens of dry goods“ (Austin 1962, 8), die schon mit der Einbettung der ,festen` Gegenstände in Prozesse Schwierigkeiten haben, zu schweigen von Prozessen als Objekten eigenen Rechts. ${ }^{3}$

An intellektueller Unattraktivität steht das spiegelbildliche Pendant zum metaphysischen Realismus diesem in nichts nach. Der metaphysische Antirealismus leugnet die Existenz einer jeden ,äußeren', vom Subjekt unabhängigen Welt. Indes bekräftigt er genau dadurch die metaphysische Teilung von Subjekt und Objekt. Der metaphysische Antirealismus entspricht in seinen heutigen Spielarten im Wesentlichen einem extremen Konstruktivismus. Seine letzte Glanzzeit feierte er in der Übernahme der Luhmann'schen Systemtheorie in Bereiche, die sich zuvor mit historistischen und hermeneutischen Positionen begnügt hatten. Überraschend ist das nicht: Die Geisteswissenschaften sind aus der Philosophie Kants entstanden und haben von Beginn an die Frage nach der Erkenntnis des Gegenstands (Epistemologie) gegenüber der Frage nach dem Gegenstand der Erkenntnis (Gnoseologie) privilegiert. Das gilt nicht zuletzt für die Hermeneutik, die, mit Peter Szondi gesprochen, nicht nach dem Gegenstand, sondern nach der Erkenntnis ihres Gegenstandes fragt (Szondi 1978, 263-264).

Zum metaphysischen Realismus und Antirealismus gibt es zwei antimetaphysische Gegenstücke, die seit einiger Zeit die Geisteswissenschaften erobert haben bzw. erobern wollen: antimetaphysischer Antirealismus und antimetaphysischer Realismus. Der antimetaphysische Antirealismus interpretiert die Welt und

2 Der metaphysische Realismus taucht immer wieder an unerwarteten Stellen auf, auch die modernste Verpackung darf nicht darüber hinwegtäuschen. Viele Ansätze etwa der Digital Humanities hängen von ihm ab. In Franco Morettis bekannter Aufsatzsammlung Distant reading etwa, die versuchte, aus dem Geist der positivistischen italienischen Philologie die US-amerikanische Kultur des close reading zu unterlaufen, werden die Studienobjekte notwendigerweise immer schon als gegeben angesehen; auch der eigene epistemologische Standpunkt wird nicht mehr problematisiert (vgl. Moretti 2013).

3 Schon in einer der wichtigsten neueren Ontologien wies Nicolai Hartmann auf den letztlich aus der alten Substanzlehre stammenden Grundfehler hin, Realität schlechthin mit räumlich bestimmter Materie zu verwechseln. Zeit sei für sie wesentlicher, da auch geistige und individuelle Prozesse an sie geknüpft seien: „Die wahren Merkmale der Realität hängen nicht an den Kategorien des Raumes und der Materie, sondern an denen der Zeit und der Individualität“ (Hartmann 1949, 22). 
ihre Gegenstände primär als Resultate epistemischer Ordnungen und kultureller Praktiken, hat aber den radikalen Konstruktivismus einerseits und traditionelle subjektzentrierte Positionen andererseits überwunden. Ihm sind verschiedene Strömungen zuzuordnen, die bisweilen unter dem Begriff des Poststrukturalismus zusammengefasst werden. Gegen diesen tritt wiederum der antimetaphysische Realismus in Erscheinung, der die Renaissance des Realismus nicht zuletzt als Reaktion auf die postmodernen Entwicklungen im antimetaphysischen Antirealismus begründet. Zentral ist hier die Heraustrennung der Ontologie aus dem alten metaphysischen Rahmenverständnis, etwa in der Akteur-Netzwerk-Theorie Bruno Latours oder den (davon z. T. abgeleiteten) Formen des spekulativen Realismus. Die gegenwärtig tonangebenden Strömungen des antimetaphysischen Realismus treten unter den jeweils programmatisch gemeinten Titeln des ,Neuen Realismus' bzw. der ,Objektorientierten Ontologie‘ auf. Beide enthalten jedoch Elemente, die sie ungeeignet erscheinen lassen, zum tieferen Verständnis der Logiken der Sammlung Entscheidendes beizutragen.

Der Neue Realismus ist die aus heterogenen Gegenbewegungen zur poststrukturalistischen Theorie geborene Wiederbelebung insbesondere klassischer deutscher Positionen, die freilich ausgerechnet den wichtigsten Vorläufer, der viele seiner Argumente vorwegnimmt, gänzlich ignoriert, nämlich den Kritischen Realismus bzw. die Neue Ontologie Nicolai Hartmanns (vgl. Gabriel 2014). ${ }^{4}$ Wie schon Hartmann bekämpft der Neue Realismus einerseits einen geistlosen Naturalismus und andererseits einen radikalen Konstruktivismus, die allerdings beide in Reinform kaum noch vertreten werden und deshalb als bloße Strohmänner herhalten müssen. Seine Innovation bestand darin, den Naturalismus nicht mehr durch den Appell an Sprachspiele, Fiktionalität und Literarizität zu sabotieren, also durch den Antirealismus, sondern durch eine alternative Form des Realismus, die eben nicht mehr metaphysisch sein soll (vgl. Gabriel 2016, 29, 33-35).

Die Verbindung zwischen Epistemologie und Ontologie leistet in der Variante Markus Gabriels der Begriff des Sinnfeldes, der gegen die Einseitigkeiten beider Orientierungen gerichtet ist und von der Abschaffung eines Begriffs der „Welt“ ausgeht, die es jeweils zu erkennen oder anzuerkennen gebe. Die „Welt“ wird als Ganzheitsvorstellung all dessen, was existiert, aus dieser Perspektive selbst als metaphysisch entlarvt und ersetzt durch die „Pluralität von Sinnfeldern [...] die

4 Im Grunde wird hier Hartmanns Grundeinsicht variiert, derzufolge die Erkenntnistheorie nicht Fundamentalphilosophie sein könne, sondern ontologischer Vorarbeit bedürfe. Schon Hartmann lehnte in seinen zahlreichen grundlegenden ontologischen Werken den metaphysischen Realismus als eine Art naiven Empirismus ab, ohne sich zugleich gegen die empirischen Wissenschaften zu wenden. 
sich prinzipiell nicht totalisieren lässt“ (Gabriel 2016, 179). Im Grunde ist diese Argumentation zirkulär, denn der Begriff des Sinnfeldes erfasst lediglich das Ausgangsdilemma. Zudem ist nicht ersichtlich, warum es nicht, was doch offensichtlich der Fall ist, Sinnfelder geben kann, für die die „Welt“ ein zentrales Element ist. Die Theorie der Sinnfelder ist weder klar bestimmt noch abgegrenzt zu alternativen Modellen diskursiver oder kultureller Praktiken. Die Bereichsontologie (vgl. Gabriel 2016, 174-176) ist vom Bereichskonstruktivismus schwer zu unterscheiden. Keine Diskurstheoretikerin würde doch behaupten, dass die Diskurse völlig unabhängig von den Gegenständen existieren, auf die sie sich beziehen. Gabriel verteidigt den ontologischen Status epistemischer Theoriekonstruktionen, insofern diese dazu dienten „Gegenstände epistemisch so zu individuieren, dass dies ihren ontischen Individuationsbedingungen entspricht“ (Gabriel 2016, 35). Aber die Herausforderung läge ja nun gerade darin, den Nachweis zu führen, dass und wie sie es tun - den bloßen Anspruch darauf erhebt auch der radikalste Konstruktivist. Im Übrigen ist vor diesem Hintergrund nicht einsichtig, warum dieser Anspruch nicht auch für den sog. gesunden Menschenverstand gelten darf, der in der Erkenntnistheorie „nichts zu suchen“ habe (Gabriel 2016, 42). Im Gegenteil reicht dieser in bestimmten Fällen doch völlig aus, um die „ontischen Individuationsbedingungen“ etwa einer Briefmarkensammlung zu bestimmen. In Nicolai Hartmanns Ontologie war die sog. natürliche Einstellung deshalb die gewissen Kontexten angemessene Position, von der die Philosophie auszugehen hat. Dem Neuen Realismus fehlt im Vergleich dazu hingegen eine Skalierungkomponente. ${ }^{5}$

Die besonders im angelsächsischen Bereich verbreitete Version des antimetaphysischen Realismus, die zunehmend auch im Bereich kontinentaler Kunstheorie rezipiert wird, bezeichnet sich selber als Objektorientierte Ontologie (mit der selbstgewählten Abkürzung 000 bzw. „Triple-O“; vgl. Harman 2018). Sie gehört in den weiten Bereich des sog. New Materialism, der im Unterschied zum poststrukturalistischen Materialitätsbegriff wieder ontologisch, z. T. sogar physikalistisch argumentiert, freilich ohne naturwissenschaftlichen Anspruch. ${ }^{6}$ Im Vergleich mit der Sinnfeld-Theorie ist die Objektorientierte Ontologie insofern radikaler, als sie jeden Anflug von Anthropozentrismus oder Privilegierung menschlicher Perspektiven aufs Schärfste bekämpft - genealogisch steht dahinter wohl noch immer die französische Rezeption von Heideggers HumanismusBrief, vermittelt in neuerer Zeit insbesondere durch die ,Demokratisierung“ der Ding-Welt bei Bruno Latour. Die deutsche Tradition idealistisch-dialektischen

5 Zur Skalierung in den Geisteswissenschaften vgl. Spoerhase (2018).

6 Das führt bisweilen zu einer fragwürdigen Begeisterung für Panpsychismus, Animismus oder Schamanismus. Zu Kritik vgl. etwa Zahavi (2016) sowie Boysen (2018). 
Denkens wird dagegen ausgeblendet. Hauptgegner ist der sog. Korrelationismus, der seit Kant angeblich die einzige Alternative zum metaphysischen Realismus blieb, nämlich die Auffassung, dass „wir Zugang nur zu einer Korrelation von Denken und Sein haben“ nie aber nur zu einer der beiden Seiten. Der spekulative Realismus möchte hinter Kant und gleichzeitig über ihn hinausgehen, um Subjektivität und Objektivität wieder getrennt zu behandeln, die Substanz wieder selbst zu denken, nicht aber die Korrelation (vgl. Meillassoux 2014, 18).

Eine grundlegende Paradoxie der Objektorientierten Ontologie besteht in dem auf der einen Seite formulierten Zentralprinzip, dass die Dinge nie direkt zugänglich seien (z. B. Harman 2018, 7), und dem Anspruch, sich philosophisch über die Vermittlungen hinwegsetzen zu können, von der sie doch schon allein in der sprachlichen Darstellung der eigenen Theorie so augenfällig abhängt. Doch sind zwischen den vier Grundformen, die sich aus der Kombination von Metaphysik und Realismus ergeben, Mischformen und Widersprüchlichkeiten in konkreten Fällen nicht unüblich, vielleicht sogar die Regel.

Ein gutes Beispiel dafür sind die Debatten um den Begriff der Materialität, die auch für die Logiken der Sammlung relevant sind. Es ist hier nicht der Ort, den Materialitätsbegriff umfassend aufzuarbeiten, ohnehin ist Unschärfe seine größte Schwäche. ${ }^{7}$ Gleichwohl lässt sich bei ihm eine begriffliche Spaltung feststellen, die letztlich der eingangs zitierten Spaltung der Sammlung in ,Gesichtspunkte‘ und ,Gegenstände‘ entspricht. In der poststrukturalistischen Theorie bezieht sich ,Materialität‘ in erster Linie auf die Unendlichkeit des Zeichenspiels und der zirkulierenden Diskurse, die jeden Versuch, der Welt habhaft zu werden, verunmöglichen. Die Einbettung der Zeichenprozesse in ,materielle، soziale Verhältnisse soll zudem jeder neoidealistischen Flucht in die reine Abstraktion einen Riegel vorschieben. Die Frage ist freilich, ob dies nicht selber Ausdruck einer rein theoretischen und damit idealistischen Konstruktion ist. In jüngster Zeit ist der Materialitätsbegriff des Poststrukturalismus denn auch vor allem aus der Perspektive der Objektorientierten Ontologie kritisiert worden:

Materialism has come to mean simply that something is historical, socially constructed, involves cultural practices, and is contingent. It has nothing to do with processes that take place in the heart of stars, suffering from cancer, or transforming fossil fuels into greenhouse gases. We wonder where the materialism in materialism is. (Bryant 2014, 2)

7 Zur Breite des Materialitätsbegriffs vgl. Samida et al. (2014), Scholz und Vedder (2018), MüllerWille (2017, 17-33); zur Kritik am Materialitätsbegriff Benne (2015). 
Daneben gibt es freilich eine zweite verbreitete Bedeutung des Materialitätsbegriffs, die sich eher auf die physische Begrenztheit bzw. physikalische Qualitäten bezieht. Er hat sich z. B. in der Editionsphilologie durchgesetzt, in der Papierqualitäten, Tintenflecke, Buchbindungen $\mathrm{u}$. ä. eine Rolle spielen. Schon mit dem ersten Aufkommen des Materialitätsbegriffs war die begriffliche Doppelung präsent. Im Kapitel „La Matérialité du Langage“ ihres theoretischen Debüts identifizierte Julia Kristeva die physische Realität etwa der menschlichen Sprechwerkzeuge ebenfalls als materialen Faktor, der freilich von der ihr viel wichtigeren Materialität auf der Ebene der Signifikation als sozialer Praxis unterschieden wurde - so wie auch das bedeutungstragende Lautbild vom bloßen Geräusch („bruit concret“) verschieden ist (vgl. Joyaux 1969, 29-50).

Die Wiederkehr des Realismusbegriffs sowie das Interesse an dieser zweiten Facette des Materialitätsbegriffs gehören eng zusammen; sie lassen sich womöglich von den materialgestützten Neuansätzen in jenen Disziplinen herleiten, die wie z. B. in der Ethnologie vom Kulturbegriff und damit den Kulturwissenschaften, d. h. nicht länger vom Geistbegriff her argumentieren (siehe z. B. Hahn 2005). Zur Debatte steht in der kulturellen Materialisierung nichts weniger als der Abschied von Paradigmen der Repräsentation, die in der Semiotik des 20. Jahrhunderts kulminierte. ${ }^{8}$ Der Materialitätsdiskurs lässt sich dergestalt als Revanche eines entscheidenden Versäumnisses schon in der Grundlegung der modernen Zeichentheorie bei Ferdinand de Saussure deuten. Das Zeichenmodell im Cours de linguistique générale war, anders als von den meisten Geisteswissenschaftlern angenommen, nicht binär, sondern ternär. Das Zeichen besteht hier nicht nur aus dem Bezeichneten und Bezeichnenden, sondern beide werden von einer „materiellen Hülle“ (enveloppe matérielle) zusammengehalten, die also Teil des Zeichens, aber selber nicht zeichenhaft ist. Der Indogermanist de Saussure stammte aus der junggrammatischen Schule, in der Lautveränderungen als rein physikalische Prozesse studiert wurden, die naturwissenschaftlichen Gesetzen unterlagen. Von Bedeutungen waren sie völlig unabhängig. Der Saussure des Cours versprach die Klärung der Frage, wie die Veränderungen der als autonom gedachten Töne im Lautwandel mit dem übrigen Sprachwandel zusammenhängt, welches Verhältnis zwischen son und mot bestehe (vgl. Saussure 1996, 194, 197). Sein Trick bestand nun darin, die Lautung von seiner Repräsentation (dem Lautbild) zu unterscheiden, das den eigentlichen Zeichenbestandteil ausmacht (vgl. Saussure 1996, 98; ausführlicher Jäger 1975; Benne 2018). Er versäumte am Ende aber, die ,materielle Hülle‘ wieder in die Theorie zu integrieren. Im neueren Interesse an Materialität und Realismus verschafft sich die Hülle des Zeichens erneut Geltung: einmal in

8 Dieser Abschnitt folgt Benne und Spoerhase (2019, 3-6). 
ihrer eigenen materiellen Hüllenhaftigkeit, aber auch in der ihr eigenen Zeichenhaftigkeit. Der Materialitätsdiskurs entsteht dort, wo die Hülle des Zeichens selber zum Zeichen wird, das aber nicht länger nur als Zeichen analysiert werden kann.

Die programmatischen Schriften des spekulativen Realismus bieten hierfür schönstes Anschauungsmaterial. Bei seinen Protagonisten handelt es sich typischerweise um abtrünnige French theorists, die in ihrem Furor eine dem von ihnen als „discursivism“ verabscheuten Denken möglichst entgegengesetzte Position besetzen wollen:

History became a history of discourses, how we talk about the world the norms and laws by which societies are organized, and practices came to signify the discursive practices through the agency of the signifier, performance, narrative, and ideology - that form subjectivities. Such a theory of society was, of course, convenient for humanities scholars who wanted to believe that the things they work with - texts - make up the most fundamental fabric of worlds and who wanted to believe that what they do and investigate is the most important of all things. (Bryant 2014, 1)

Bryant, der sich explizit den Eifer des Konvertiten bescheinigt, geht von der Einsicht aus, ,that the signifier, meaning, belief, and so on are not the sole agencies structuring social relations“ (Bryant 2014, 4-5). Das aber hatte auch keiner der Diskursbegründer je behauptet. Die entsprechende Trotzreaktion, die bewusst ihrer Sehnsucht nach „stuff“ und Realität Ausdruck verleiht, endet doch ungewollt wieder im metaphysischen Realismus, solange sie sich nicht dem Problem der eigenen Beschreibung dieses „stuff“ stellt. ${ }^{9}$ Das ist überhaupt kein neues Problem; und es ist erst recht keine abwegige Forderung schwärmerischer Kontinentalphilosophen: Der Anspruch auf ,Realismus ‘ war schon immer ein Kampfbegriff, der einen vermeintlichen Gegensatz voraussetzte, der nur relativ zu jenem existiert. ${ }^{10}$

9 „In describing my position as unabashedly naïve, I only mean to say that the world is composed of physical things such as trees, rocks, planets, stars, wombats, and automobiles, that thought and concepts only exist in brains, on paper, and in computer data banks, and that ideas can only be transmitted through physical media such as fiber optic cables, smoke signals, oxygen-rich atmospheres, and so on“ (Bryant 2014, 6). Bei Bryant ist nicht nur die ältere kritische Ontologie seit Hartmann abwesend, sondern auch die neueren ontologisch ausgerichteten Medientheorien etwa Friedrich Kittlers oder die Theorie der Affordanzen seit J.J. Gibson.

10 Siehe schon Austin: „It should be quite clear, then, that there are no criteria to be laid down in general for distinguishing the real from the not real. How this is to be done must depend on what it is with respect to which the problem arises in particular cases“ (Austin 1962, 76). Meinem Eindruck nach, aber das muss hier spekulative Vermutung bleiben, verbindet sich die unverhohlen naive Liebe zum „stuff“ und die damit einhergehende Linné’sche Sammel- und Kategorisie- 
Das Problem das Neuen Realismus wie auch des Spekulativen Realismus im Sinne der Objektorientierten Ontologie ist, allen gegenteiligen Beteuerungen zum Trotz, paradoxerweise die erkenntnistheoretische Verengung des Realismus. Im rein ,realistischen‘ Zugriff auf die Phänomene gehen jene Aspekte verloren, die gerade für eine Logik der Sammlung zentral wären: das Begehren nach den Gegenständen, ihre Performanz, die offenen Möglichkeiten eines je neuen Umgangs mit ihnen. Was Klaus Kastberger, Stefan Maurer und Christian Neuhuber in ihrer Einleitung eines anderen Bandes dieser Reihe zum „Schauplatz Archiv“ hervorheben, nämlich die Dimensionen der Phantasmagorie, des Simulakrums und der Inszenierung von Archiven (vgl. Kastberger et al. 2019, 8), gilt in hohem Maße auch für den „Schauplatz“ der Sammlung. Sammlungen sind nur ein Spezialfall jener ,Objekte‘, die unabhängig von ,Subjekten` zu studieren zwar möglich, aber sinnlos wäre. Es liegt auf der Hand, dass wir wesentliche Aspekte übersehen, wenn wir Damien Hirsts eingelegte Kuh nur als physische Kuh untersuchen; zu ihrer ,Realität‘ gehört der Kontext der Ausstellung genauso dazu wie die Beziehung der Ausstellungsbesucher zu seinem Kunstwerkcharakter. Es bedarf deshalb einer theoretischen Rahmung, die den naiven Realismus nicht nur dem Lippenbekenntnis nach verlassen hat, ohne dabei freilich die Ambition aufzugeben, sich mit ,Realität‘ zu befassen. Um des realistischen Anspruchs willen muss eine Logik der Sammlung die Gegenstände der Sammlung in Bezug auf ihre Relationalität und die Praktiken ihrer Entstehung und Verwendung studieren, aber eben nicht ausschließlich darauf. Man muss sich eben nicht, wie manche neue oder spekulative Realisten insinuieren, zwischen Epistemologie und Ontologie entscheiden.

\section{Gegenständlichkeit als Logik der Sammlung}

Ich möchte aus den genannten Gründen vorschlagen, für die Frage nach den Logiken der Sammlung auf das Denken der Gegenständlichkeit zurückzugreifen. Diese lange in Vergessenheit geratene Philosophie wurde in der Zwischenkriegszeit entwickelt; ihre Rezeption kam aber durch die nationalsozialistische Verfolgung einiger Protagonisten wie so viele andere fruchtbare Entwicklungen dieser Ära an ein abruptes Ende. Auf den Höhepunkt des Neukantianismus waren in der späten Weimarer Zeit Versuche gefolgt, epistemologische mit ontologischen und

rungswut der „neuen Archivisten“ (Moritz Baßler) häufig mit einem nostalgischen Grundgestus. Bisweilen erscheint der ,neue Realismus‘ deshalb, gewiss gegen seine Intentionen, als Sehnsucht nach einer guten alten Übersichtlichkeit. 
phänomenologischen Perspektiven zusammenzudenken. Heideggers Fundamentalontologie überschattete sie in der Nachkriegszeit. Die jüngste Hinwendung zum Realismus bietet die Chance, sie wiederzuentdecken und weiterzuentwickeln.

Ins Zentrum dieser Wiederentdeckung möchte ich den deutsch-österreichischen Meinong-Schüler Richard Hönigswald (1875-1947) stellen, an dessen Vertreibung und Vergessen Heidegger selbst aktiven Anteil hatte und der von der Fachphilosophie heute bis auf wenige Ausnahmen ignoriert wird. ${ }^{11}$

Bei dem naturwissenschaftlich, ontologisch und phänomenologisch geschulten Kantianer Hönigswald wird der Gegenstand unabhängig vom Subjekt gedacht, das als (historisch veränderliche) „Monas“ in der Erlebnisrelation zu ihm gleichwohl konstitutiv für ihn ist. Die Monas agiert zugleich unter der Bedingung der Selbstpräsenz (ist sich selbst Gegenstand). „Gegenständlichkeit“ ist bei Hönigswald Bedingung dafür, dass sich ein (individuelles oder kollektives) Ich überhaupt auf einen Gegenstand richten kann. Sie enthält deshalb auch immer das sich dem Zugriff Entziehende, das Entgegenstehende seiner Eigenlogik. Da zur Gegenständlichkeit auch die Bedingungen seiner Möglichkeit sowie die Erlebnisrelationen gehören, die in sich selbst historisch-kulturell veränderlich sind, werden Praktiken der Identifizierung und Aneignung der verschiedenen Arten von Gegenständen zu seinem Apriori. Gleichzeitig wird die Gegenständlichkeit als ausgehend vom Gegenstand selbst gedacht, der, in heutiger Terminologie, Affordanzen bietet, denen womöglich noch keine existierenden Praktiken entsprechen, derer sich aber ältere Praktiken modifizierend annehmen können oder aus denen ganz neue Praktiken entstehen mögen.

,Gegenständlichkeit‘ enthält damit sowohl die Reflexion und Bestimmung der Gegenstände, ausgehend von ihnen selbst, wie auch ihre Wirkungen. Dass uns die Gegenstände in der Erlebnisrelation vermittelt entgegentreten (ohne schlicht von uns konstruiert zu sein), stellt keineswegs ihre Eigenlogik infrage. Vielmehr geht es in der Philosophie der Gegenständlichkeit ja gerade um die Frage, inwieweit unser Erleben oder unsere Bestimmungen von ihnen beeinflusst sind. Hinter dem Erleben verbirgt sich keine Neuauflage des Psychologismus, sondern die Anerkennung der Präsenz als Vollzug des Erlebens, die ein Teil der Beziehung zwischen Gegenstand und Monas ist. ${ }^{12}$

11 In der Heidegger-Nachfolge taucht Hönigswald trotz der Appropriation seines Zentralbegriffes bis heute nicht auf (vgl. Figal 2006). Seit den 1990er-Jahren setzte eine vorsichtige Wiederentdeckung Hönigswalds ein (vgl. z. B. Schmied-Kowarzik 1997), die freilich von der gleichzeitig einsetzenden Renaissance der Philosophie Ernst Cassirers überflügelt wurde.

12 Siehe insbesondere Hönigswald (1997). Aufgegriffen und für literaturtheoretische Fragestellungen weiterentwickelt in Benne (2015). 
Dass die Logik der Sammlung aus ihrer Gegenständlichkeit zu rekonstruieren ist, bedeutet, dass ein Verständnis jeder Sammlung vom Verständnis nicht nur der in ihr enthaltenen Gegenstände oder der Überzeugungen, Schematisierungen und Praktiken abhängig ist, aufgrund derer sie zustande gekommen ist, sondern von einer möglichst genauen Analyse und Beschreibung der Relationen zwischen Sammlungsgegenständen und -kontexten, Sammlern und Sammlungsnutzern. Das klingt banal, ist es aber angesichts des theoretischen Diskussionsstandes nicht. Das gegenständliche Denken ist deskriptiv im Sinne des Eingeständnisses, dass jedes Beschreiben auch ein Erschreiben ist. Die sprachlich-konzeptuelle Reflexion der Gegenstände geht von (historisch veränderlichen) Affordanzen der Gegenstände aus, die durchaus auch präreflexiver (d. h. nicht automatisch diskursiver) Natur sein können. Sie wird durch die Vermittlung mit unseren vorgeprägten Einteilungen der Gegenstände zur Gegenständlichkeit, die allein das Vermögen hat, diese Einteilungen zu verändern. Gegenständlichkeit ist in diesem Sinne auch ein historisches und skalierbares Phänomen. Die Gegenständlichkeit einer privaten Schallplattensammlung ist gewiss einfacher zu beschreiben als eine Sammlung zeitgenössischer Medieninstallationen. Für die eigene Schallplattensammlung reicht ein vortheoretisches Sammlungsmodell und damit ein metaphysischer Realismus aus, für die Medienkunst ist schon die Frage nach der Abgrenzung und Bestimmung der Gegenstände Teil der Erlebnisrelation.

Für die Logik der Sammlung bietet die Gegenständlichkeitsphilosophie zwei weitere Vorteile. Erstens löst sie die Gegenstände, auf die sie sich bezieht, nie völlig in der Beschreibung, den Praktiken oder den Affordanzen auf. Sie erkennt das ,Entgegenstehende“ der Eigenlogik an, das deshalb immer wieder neuer Beund Erschreibungen bedarf. Weil sie, zweitens, von der grundlegenden Veränderlichkeit der Erlebnisrelationen ausgeht, die Auswirkung auf unser Selbstverständnis, aber auch auf das Verständnis der Gegenstände selbst hat, reduziert sie Sammlungen nicht auf ihre Urheber. Aus gegenständlicher Sicht ergibt sich die Logik der Sammlung immer in einem Hier und Jetzt der Begegnung. Eine Sammlung, die irgendwann auf vortheoretischem Wege zustande kam, mag plötzlich in neuem Licht erscheinen. Man denke an die Aura historischer Sammlungen und Kuriositätenkabinette, in denen nicht nur historische Gegenständlichkeiten konserviert werden, sondern die unbewusst auch Gegenständlichkeiten enthält, die sich erst späteren Betrachtern eröffnen, die jenseits der Sammlerintention neue Bezüge oder sogar neue Gegenstände in ihnen entdecken. Deshalb ist die Bewahrung und kulturwissenschaftliche Aufbereitung historischer Sammlungen $\mathrm{zu}$ einem zentralen Forschungsfeld geworden. An der Gegenständlichkeit der Sammlung entscheidet sich die Historizität ihrer Logik, die zugleich von ihrer Integrität auszugehen hat. 


\section{Abschließende Rindbemerkungen}

Kehren wir zum Ausgangspunkt unserer vorgestellten Rinderherde zurück. Eine Kuh ist niemals nur eine Kuh. Die ,reale‘ Kuh als diskrete Einheit ist selber nur eine Abstraktion, eine Phantasmagorie von ,Realisten‘. In jedem möglichen und tatsächlich realen Szenario ist die Kuh in eine Fülle von sowohl physischen wie diskursiven Beziehungen eingebettet, die zudem dergestalt miteinander verquickt sind, dass jede Identifizierung der Kuh als Kuh nur der Anfang einer Fülle von Bestimmungen sein kann. Ähnlich verhält es sich mit Sammlungen und ihrer Gegenständlichkeit. Die Sammlung unterscheidet sich von anderen Gegenständen lediglich durch eine spezifische innere Beziehung und Staffelung ihrer Elemente, wobei es dafür eine unendliche Vielzahl von Möglichkeiten und deshalb realen wie möglichen Sammlungen gibt. Wir könnten unsere Auffassung der Gegenständlichkeit von Sammlungen zwar auf eine Weise restringieren, die ausschließt, Kuhherden als Sammlungen aufzufassen. Aber die auf jeden Einzelfall anwendbare Universaldefinition liegt gar nicht im Interesse des Gegenständlichkeitsdenkens. Ich bin sicher, dass man mit Hilfe von Gabriels Sinnfeldtheorie und wohl auch mit einer intelligent weitergedachten Objektorientierten Ontologie zu ähnlichen Ergebnissen gelangt. Bei Harman ist das Objekt immer mehr als seine Bestandteile, aber weniger als seine Effekte (vgl. Harman 2018, 53). Das gilt gewiss auch für Sammlungen, besser: So wie jede Sammlung selbst ein Objekt eigenen Rechts ist, wäre jedes Objekt auch eine Sammlung. Allerdings müsste man sich dann noch von einer dogmatischen Auffassung der flat ontology verabschieden.

Die Frage, worin denn die Gegenständlichkeit von Damien Hirsts Mother and Child, Divided liege, ist genauso sinnlos wie die Frage, ob es sich dabei um Kühe handele. Der Durchbruch Damien Hirsts in den 1990er-Jahren geschah zu einer Zeit, als der von den Neuen Realisten kritisierte ,Diskursivismus‘ seinen vorläufigen Höhepunkt erlebte. Gefördert wurde Hirst zudem von einer erfolgreichen Werbeagentur, die durch das Spiel mit Zeichen reich geworden war. In die poststrukturalistischen Gewissheiten schlug die Sammlung und Ausstellung ,realer toter Tiere ein wie eine Bombe. Möglicherweise war dies überhaupt die Initialzündung für das neue Interesse an ,Realität‘, das dann freilich selbst zu einem werbetechnisch ausschlachtbaren Phantasma wurde, wie es in der virtuellen Authentizitätspose der Hipster- und Instagramkultur kulminierte, in der ebenfalls v. a. vermeintlich authentische Momente eines ,realen` Lebens gesammelt werden.

Hirsts tote Tiere (darunter auch Haie und Schafe) ließen Einblicke in das Innenleben der Objekte zu, die den realistischen Blick in Form eines Hyperrealismus radikalisierten und damit doch wieder diskursiv aufbrachen. Ich selber sah Mother and Child, Divided zuerst in der Wanderausstellung Sensation, die nicht 
nur zu einem Meilenstein der jüngeren britischen Kunstszene wurde, sondern auch für ihre kommerziellen Züge stark in Kritik geriet. Die Frage, ob ich Hirsts Werk primär als Teil einer Sammlung, einer konkreten Ausstellung oder aber als individuellen Ausdruck analysiere, hat weitreichende Konsequenzen. Die Aufgabe der Kunst ist es seit jeher gewesen, die auf Gegenständlichkeiten bezogenen Konventionen $\mathrm{zu}$ erweitern oder $\mathrm{zu}$ verengen. Ein ,realistischer` Blick auf Mother and Child, Divided muss die Realität des Kunstwerkes über die Kontextualisierung in der Sammlung hinaus einbeziehen, seien es die Anklänge an ein barockes memento mori, sei es die Wahl des Sujets, in der sich auch eine basale menschliche Beziehung ausdrückt, sei es die Aufstellung in Glaskästen, die zur multiperspektivischen Erkundung auffordert und $\mathrm{zu}$ der Praktik der Kunstbetrachtung, die zwischen zweidimensionaler Bildbetrachtung und dreidimensionalem Skulpturenrundgang unterscheidet, eine neue Variante hinzufügte, in dem sie die Zwei- und Dreidimensionalität als Kippbild gestaltete. Sammlungen als Ganzes funktionieren auf ähnliche Weise - und das gilt beileibe nicht nur für Kunstsammlungen. Wo sie ihre eigene Gegenständlichkeit reflektieren, tragen sie dazu bei, dass wir Gegenstände neu zu bestimmen lernen - und erst dadurch verstehen, was eigentlich gesammelt wurde. So verändert sich von der Gegenständlichkeit der Sammelobjekte her die Gegenständlichkeit der Sammlung selbst. Darin zeigt sich nicht nur ihre Logik, sondern auch ihre Ethik.

\section{Literaturverzeichnis}

Austin, J. L. Sense and Sensibilia. Reconstructed from the manuscript notes by G. J. Warnock. Oxford: Clarendon, 1962.

Benne, Christian: Die Erfindung des Manuskripts. Zur Theorie und Geschichte literarischer Gegenständlichkeit. Berlin: Suhrkamp, 2015.

Benne, Christian. „Apologie des Buchstaben A. Über Indogermanistik und Moderne“. Strukturalismus, heute. Brüche, Spuren, Kontinuitäten. Hg. Martin Endres und Leonhard Herrmann. Stuttgart: Metzler, 2018. 29-43.

Benne, Christian, und Carlos Spoerhase: „Materialität: von Blättern und Seiten. Einleitung“. Kodex. Jahrbuch der Internationalen Buchwissenschaftlichen Gesellschaft 2019: 3-6.

Boysen, Benjamin. „The Embarrassment of Being Human: A Critique of New Materialism and Object-Oriented Ontology“. Orbis litterarum 73.3 (2018): 225-242.

Bryant, Levi R. Onto-Cartography. An Ontology of Machines and Media. Edinburgh: Edinburgh Univ. Press, 2014.

Deutsches Wörterbuch von Jacob u. Wilhelm Grimm. 16 Bde. in 32 Tlbde. Leipzig 1854-1961. URL: http://www.woerterbuchnetz.de/DWB (Lemma: „Sammlung“).

Figal, Günter. Gegenständlichkeit. Das Hermeneutische und die Philosophie. Tübingen: Mohr-Siebeck, 2006.

Gabriel, Markus (Hg.). Der neue Realismus. Berlin: Suhrkamp, 2014. 
Gabriel, Markus. Sinn und Existenz. Eine realistische Ontologie. Berlin: Suhrkamp, 2016. Hahn, Hans Peter. Materielle Kultur. Eine Einführung. Berlin: Reimer, 2005.

Harman, Graham. Object-Oriented Ontology. A New Theory of Everything. Harmondsworth: Penguin, 2018.

Hartmann, Nicolai. Neue Wege der Ontologie. 3. Aufl. Stuttgart: Kohlhammer, 1949.

Heidegger, Martin. „Heraklit. Logos. Heraklits Lehre vom Logos“. Gesamtausgabe (GA). Bd. 55. Frankfurt a. M.: Klostermann, $1975 \mathrm{ff}$.

Heidegger, Martin. „Was heißt Lesen?“. Gesamtausgabe (GA). Bd. 13. Frankfurt a. M.: Klostermann, 1975ff. 111.

Hönigswald, Richard. Grundfragen der Erkenntnistheorie. Hg. Wolfdietrich Schmied-Kowarzik. Hamburg: Meiner, 1997.

Jäger, Ludwig. Zu einer historischen Rekonstruktion der authentischen Sprach-Idee F. de Saussures. Düsseldorf, Univ., Diss. 1975.

Joyaux [Kristeva], Julia. „La Matérialité du Langage”. Le langage, cet inconnu. Paris: S.G.P.P., 1969. 29-50.

Kastberger, Klaus, Stefan Maurer und Christian Neuhuber (Hg.). Schauplatz Archiv. Objekt Narrativ - Performanz. Berlin, Boston: de Gruyter, 2019.

Meillassoux, Quentin. Nach der Endlichkeit. Versuch über die Notwendigkeit der Kontingenz. Übers. v. Roland Frommel. Zürich: Diaphanes, 2014.

Moretti, Franco. Distant reading. London, New York: Verso, 2013.

Müller-Wille, Klaus. Sezierte Bücher. Hans Christian Andersens Materialästhetik. Paderborn: Fink, 2017.

Samida, Stefanie, Manfred K. H. Eggert, und Hans Peter Hahn (Hg.). Handbuch Materielle Kultur: Bedeutungen, Konzepte, Disziplinen. Stuttgart: Metzler, 2014.

Saussure, Ferdinand de. Cours de linguistique générale. Hg. Tullio de Mauro. Paris: Fayard, 1996.

Schmied-Kowarzik, Wolfdietrich (Hg.). Erkennen - Monas - Sprache. Internationales RichardHönigswald-Symposion Kassel 1995. Würzburg: Königshausen und Neumann, 1997.

Skirl, Miguel, Andreas Urs Sommer und Dagmar Winter. Die Hortung. Eine Philosophie des Sammelns. Düsseldorf: Parerga, 2000.

Scholz, Susanne, und Ulrike Vedder (Hg.). Handbuch Literatur \& Materielle Kultur. Berlin, Boston: de Gruyter, 2018.

Sommer, Manfred. Sammeln. Ein philosophischer Versuch. Frankfurt a. M.: Suhrkamp, 1999.

Spoerhase, Carlos. Das Format der Literatur: Praktiken materieller Textualität zwischen 1740 und 1830. Göttingen: Wallstein, 2018.

Stagl, Justin: „Homo Collector. Zur Anthropologie und Soziologie des Sammelns“. Sammler - Bibliophile - Exzentriker. Hg. Aleida Assmann, Monika Gomille und Gabriele Rippl. Tübingen: Narr, 1998. 37-54.

Szondi, Peter. „Über philologische Erkenntnis“. Schriften I. Hg. Jean Bollack et al. Frankfurt a. M.: Suhrkamp, 1978. 263-286.

Zahavi, Dan. „The End of What? Phenomenology vs. Speculative Realism“. International Journal of Philosophical Studies 24.3 (2016): 289-309. 